\title{
Ein neues Fragment des Midrasch Bereschit Rabba
}

\author{
Andreas Lehnardt
}

U nter den in Archiven und Bibliotheken erhaltenen Einband- und Makulaturfragmenten bilden die auf Hebräisch beschrifteten Reste jüdischer Handschriften eine besondere Gruppe. ${ }^{.}$Die Erforschung dieser jüdischen Fragmente erfährt seit einigen Jahren in Deutschland und auch in benachbarten europäischen Ländern zunehmende Beachtung. ${ }^{2}$ Dennoch sind viele Funde noch unbekannt und konnten daher etwa auch im Rahmen größerer Editionsprojekte nicht berücksichtigt werden. Ihre systematische Erfassung stellt daher nach wie vor eine wichtige Aufgabe dar und wird auch in den kommenden Jahren immer wieder interessante Entdeckungen auch an entlegenen Orten zu Tage fördern.

Das hier vorgestellte Blatt wurde unlängst in der Universitätsbibliothek Frankfurt entdeckt und bietet einen wichtigen Textzeugen eines bekannten rabbinischen Bibelkommentars. ${ }^{3}$ Das Fragment trägt nun die Signatur Hebr. Fragm. V 2 und dient als Umschlag eines Sammelbandes mit der Signatur N. Libri. Ff I0739. Er enthält drei bekannte lateinische Drucke: I. Rodericus (Sancius de Arevalo), Speculum humanae vitae quo discuntiuntur [...], Argentorati [Regensburg]: Zetznerus, $16066^{4}$; 2. Caspar Schoppe, Priapeia sive Diversorum Poetarum in Priapum Lusus, Frankfurt am Main: Nebenius, Richter I506 [I606]'; 3. Gaius Valerius Catull, Casta Carmina, Frankfurt am Main: Nebenius, Richter I506 [1606]. ${ }^{6}$

Die Wiederverwendung von hebräischen Handschriften als Einbandmaterial in Frankfurter Beständen ist seit einiger Zeit genauer untersucht und kann sehr wahrscheinlich mit der Plünderung der Frankfurter Judengasse im Verlauf des so genannten Fettmilch-Pogroms (1614-1616) in Verbindung gebracht werden. ${ }^{7}$ In der Folge der Vertreibung der Juden aus der Reichsstadt gelangten zahlreiche alte jüdische Handschriften auf Messen und Märkte und wurden weit über die Grenzen der Stadt hinaus verkauft. ${ }^{8}$ Buchbindern dienten sie als willkommenes Arbeitsmaterial, ohne dass sie auf den Inhalt und Provenienz achteten - ganz so wie bei lateinischen und deutschen Handschriften. ${ }^{9}$

Das Blatt wurde querstehend als äußerer Umschlag des Sammelbandes über die Buchdeckel geklebt. Dies entspricht einer an Frankfurter Einbänden aus der zweiten Hälfte des 17. Jahrhunderts häufig zu beobachtenden Technik. Der lesbare hebräisch-aramäische Text des Fragmentes stammt aus dem spätantiken rabbinischen Kommentar zum Buch Genesis, der Teil des Midrasch Rabba, der "großer Midrasch“ genannten Sammlung exegetischer Kommentare zu den fünf Büchern Mose sowie zu den fünf Festtagsrollen (Megillot). Dieser Midrash zum Buch Genesis wurde im 5. Jahrhundert n. d. Z. redigiert und ist zu großen Teilen auf Aramäisch verfasst. Die älteste erhaltene Handschrift dieses Werkes (Ms Vatikan 6o) stammt aus dem Io. Jahrhundert. Die Anzahl der erhaltenen vollständigen Handschriften und Fragmente belegen seine große Beliebtheit und Verbreitung.

In den vergangenen Jahren wurden von mir bereits einige andere Fragmente dieses bedeutenden Werkes in Einbänden entdeckt, darunter auch eines im Frankfurter Institut für Stadtgeschichte. ${ }^{\text {Io }}$ Ein weiteres Exemplar - wiederum aus einem 
anderen Codex - konnte ich in einem Einband in der Thüringer Landes- und Universitätsbibliothek Jena identifizieren. ${ }^{\text {II } Z u l e t z t ~ f a n d e n ~ s i c h ~ a u c h ~}$ in der Staats- und Universitätsbibliothek Bremen zwei vergleichbare Fragmente; sie gilt es im Hinblick auf den neuen Fund genauer zu beachten. ${ }^{12}$

Das Frankfurter Fragment misst ca. $17 \times 14 \mathrm{~cm}$ und bietet die unteren Abschnitte zweier rechtsbündig geschriebener Kolumnen à II bzw. I2 Zeilen. Die oberen Enden der Spalten sind abgeschnitten, die unteren sind mit einem breiten Rand erhalten. Ursprünglich hatten die Spalten vermutlich ca. 29-30 Zeilen. Am oberen Rand von Kolumne b, auf dem Buchrücken, sind einige hebräische Wörter von einem alten Titelschild verdeckt. Auf dem hinteren Deckel ist noch gut die Liniierung erkennbar. Wie in aschkenasischen Manuskripten ab dem I3. Jahrhundert üblich, steht die untere Linie frei. Die leicht gerundeten quadratischen Buchstaben sind an diese Hilfslinien angehängt. Die verwendete aschkenasische Schrift weist Gemeinsamkeiten mit Schrifttypen des I3. Jahrhunderts auf. ${ }^{13}$ Charakteristisch sind etwa die Buchstaben alef, 'ayin und shin. Auffällig ist der untere Schaft des 'ayin und des peh, der wie auf älteren Inschriften und in Manuskripten des I2./13. Jahrhunderts unter den folgenden Buchstaben ausgezogen ist. Der mittlere Schaft des Buchstabens shin ist nicht bis zum Bauch des unteren geschwungenen Striches durchgezogen. Auch dies ist typisch für aschkenasische Handschriften aus dem I3. Jahrhundert. Die Abbreviaturzeichen sind als kleine Punkte über die Buchstaben gesetzt. ${ }^{14} \mathrm{Am}$ Ende von Zeile 2 in Kolumne $\mathrm{b}$ ist ein Dehnungsbuchstabe verwendet. Zwischen Sinnabschnitten ist ein Freiraum belassen.

Der erhaltene Text stammt aus Parascha 28 (bzw. 26 in Ms Vatikan, Biblioteca Apostolica, ebr. 6o) des Midrasch Bereschit Rabba. ${ }^{15}$ Die variae lectiones legen die Vermutung nahe, dass es sich um die Kopie einer älteren aschkenasischen Vorlage handelt, in der z.B. Lehnwörter (Kolumne a, Zeile 9: palatin = Palast) und Rabbinennamen (Kolumne a, Zeile 4) korrekter und vollständiger als etwa in der gedruckten Fassung tradiert waren. Die Variationen sind sprachlich jedoch nicht so alt wie in den ältesten Fragmenten aus der Kairoer Genisa oder in Ms Vatican, ebr. 60 aus dem Io. Jahrhundert. Interessant ist die Variante in dem Königsgleichnis Kolumne a, Zeile 8-ıo, wo „Fürst“ statt König (wie in Ms Vatikan u.a.) steht. Bemerkenswert ist auch ein kurzer Kommentar (perusch) am linken Spaltenrand von Kolumne b, in dem das lateinisch-griechische Lehnwort „gastriot“, Kriegslager (von Castrum?), ${ }^{16}$ erläutert wird. ${ }^{17}$ Der Kommentar ist nicht identisch mit dem Raschi-Kommentar zur Stelle nach den gedruckten Ausgaben.

Vergleicht man die paläographischen und inhaltlichen Charakteristika dieses Fragments mit den beiden von mir in der UB Bremen identifizierten Stücken, so deutet vieles darauf hin, dass es sich um Blätter aus ein und demselben Codex handelt. Sowohl die beschriebenen paläographischen Merkmale - Liniierung, Buchstabenformen, Abkürzungszeichen - als auch das rekonstruierbare ursprüngliche Seitenformat des Frankfurter Manuskripts machen es wahrscheinlich, dass die Blätter einst Teil einer Handschrift waren und dann zerschnitten zu Bucheinbänden recycelt wurden. ${ }^{18}$

Insgesamt ist der hier vorgestellte Fund also von besonderer Bedeutung, da er ermöglicht, weit verstreute Fragmente einer einst umfangreicheren Handschrift einander zuzuordnen - das Puzzle gewissermaßen um ein Stück zu ergänzen. Wie bereits in Bezug auf die Bremer Fragmente vermutet, dürften alle drei Fragmente nun sicher einen Frankfurter Bezug aufweisen. Vermutlich wurden der größere Codex, dem sie entnommen sind, dort nach dem Fettmilch-Pogrom entwendet und die Blätter dann einzeln zu Buchumschlägen umfunktioniert. ${ }^{19}$ 
Frankfurt a. M., UB, Hebr. Fragm. V 2

Abürzungen der verglichenen Textzeugen:

$T=$ Editio princeps, Venedig 1545

$\mathrm{I}=$ Vatikan, Biblioteca Apostolica. Ms 30, B1. 48r

$\zeta=$ London, British Museum, Add. 27169

$\Xi$ = Paris, BNF, Ms I49
Vgl. die wissenschaftliche Ausgabe von J. Theodor / Ch. Albeck (Hrsg.), Bereschit Rabba mit kritischem Apparat und Kommentar, Bd. I, 2. Aufl., Jerusalem 1965, S. 260-26I, Zeile 2-Zeile 2. Für eine deutsche Übersetzung mit Abweichungen vgl. August Wünsche, Der Midrasch Bereschit Rabba. Das ist die Haggadische Auslegung der Genesis, Leipzig 188I, Nachdruck Hildesheim u.a. 1993, S. I23.

Kolumne b

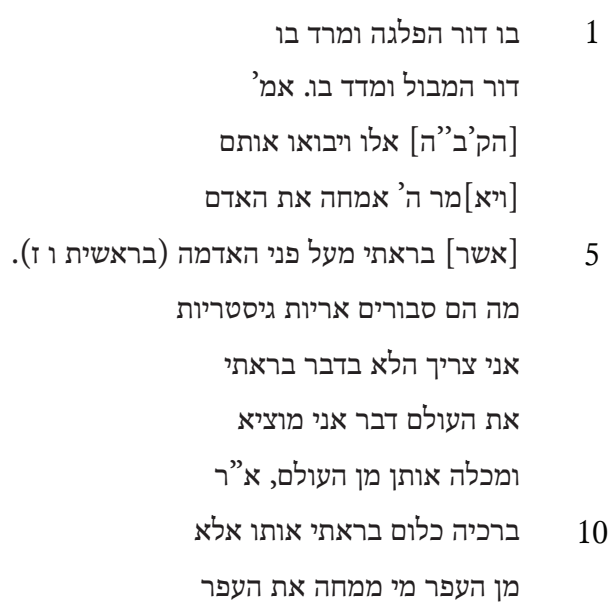

Kolumne a

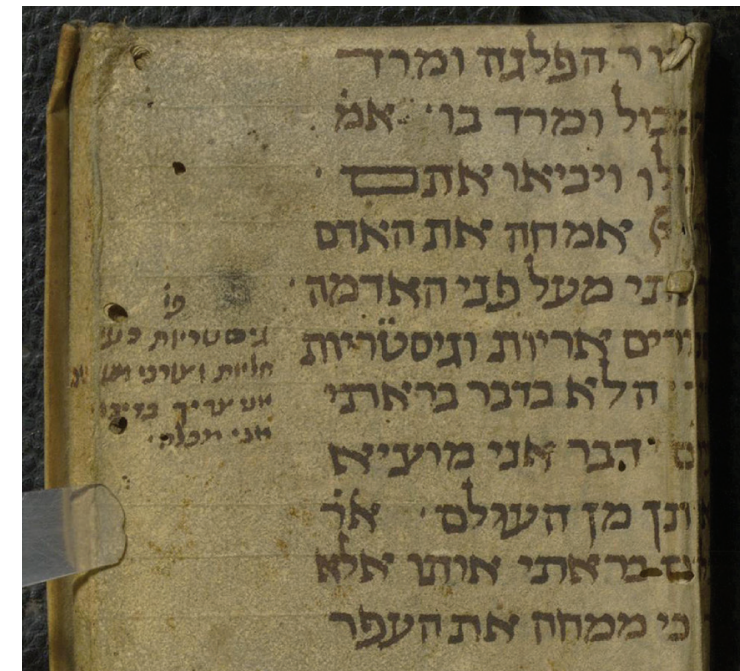

Abb. I: Frankfurt a. M., UB, Hebr. Fragm. V 2, Kolumne b (hinterer Einbanddeckel)

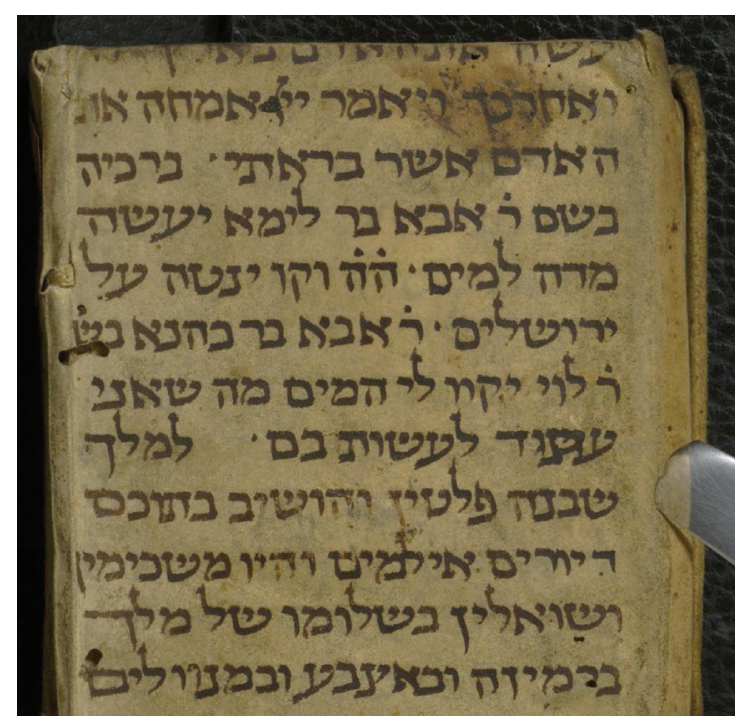

Abb. 2: Frankfurt a. M., UB, Hebr. Fragm. V 2, Kolumne a (vorderer Einbanddeckel)

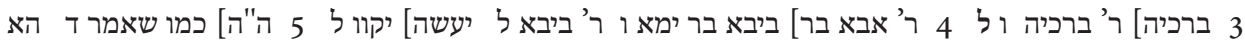

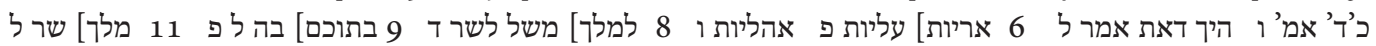




\section{Kontakt}

Andreas Lehnardt

Johannes Gutenberg-Universität Mainz · Judaistik · Saarstr. 2I · 55099 Mainz

E-Mail: lehnardt@uni-mainz.de

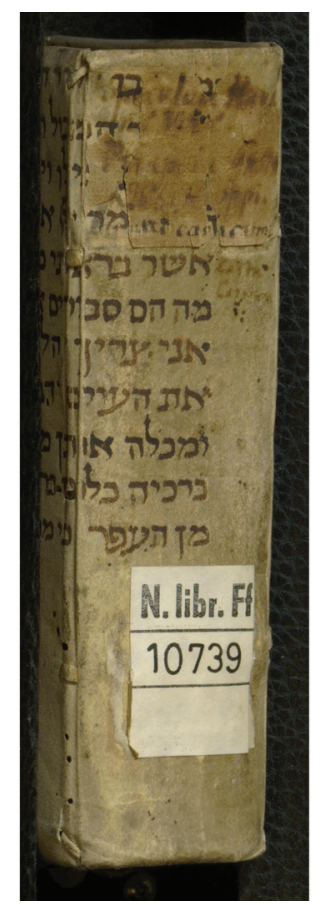

Abb. 3: Frankfurt a. M., UB,

Hebr. Fragm. V 2, Kolumne b (Buchrücken)

\section{Anmerkungen}

I Vgl. dazu Andreas Lehnardt, Hebräische Handschriften: Die hebräischen und aramäischen Einbandfragmente in deutschen Archiven, Bibliotheken und Sammlungen (Verzeichnis der Orientalischen Handschriften in Deutschland VI,2), Stuttgart 2O2I.

2 Vgl. Andreas Lehnardt (Hg.), European Genizah. Newly Discovered Hebrew Binding Fragments in Context (European Genizah. Texts and Studies 5), Leiden/Boston 2020.

3 Für den Hinweis auf diesen Fund danke ich Raschida Mansour, UB Frankfurt, Handschriftenabteilung.

4 VDi7 3:60994IS.

5 VDi7 23:27948IE.

6 VDi7 23:297054K.

7 Zur Rekonstruktion der Vorgänge vgl. Andreas Lehnardt, Hebräische Einbandfragmente in Frankfurt am Main. Mittelalterliche jüdische Handschriftenreste in ihrem geschichtlichen Kontext (Frankfurter Bibliotheksschriften II), Frankfurt a. M. 20II.

8 Vgl. dazu Andreas Lehnardt, Katalog der hebräischen Einbandfragmente in der Forschungsbibliothek Gotha. Aus den Sammlungen der Herzog von Sachsen-Coburg und Gothasschen Stiftung für Kunst und Wissenschaft (Die Handschriften der Forschungsbibliothek Gotha 4), Wiesbaden 20I9, S. XXIX. 
9 Vgl. dazu Andreas Lehnardt, Hebräische Handschriftenfragmente im Blick der judaistischen Forschung, in: Fragment und Makulatur. Überlieferungsstörungen und Forschungsbedarf bei Kulturgut in Archiven und Bibliotheken, hg. von Hans Peter Neuheuser und Wolfgang Schmitz (Buchwissenschaftliche Beiträge 9I), Wiesbaden 20I5, S. $19 \mathrm{I}-207$

Io Vgl. Lehnardt [Anm. 7], S. 153-155.

II Andreas Lehnardt, Midrash Bereshit Rabbah in Christian Bindings: A Newly Discovered Medieval Ashkenazic Manuscript Fragment from Jena, in: Hebrew Texts in Jewish, Christian and Muslim Surroundings, hg. von Klaas Spronk und Eveline Staalduine-Sulman (Studia Semitica Neerlandica 69), Leiden/Boston 20I8, S. 170-187.

12 Andreas Lehnardt, Two New Fragments of Midrash Bereshit Rabbah in the State and University Library Bremen, in: European Genizah [Anm. 2], S. IO7-II6.

13 Vgl. Edna Engel, Calamus or Chisel. On the History of the Ashkenazic Script, in: 'Genizat Germania' - Hebrew and Aramaic Binding Fragments from Germany in Context, hg. von Andreas Lehnardt (European Genizah. Texts and Studies I), Leiden/Boston 2010, S. I83-197, hier S. I86 (Plate 2) und S. I89. Vgl. auch Edna Engel, Remarks on the Ashkenazic Script, in: Specimens of Medieval Hebrew Scripts. Vol. III: Ashkenazic Script, hg. Von Edna Engel und Malachi Beit-Arié, Jerusalem 2017, S. XXIXf.

14 So auch in den Bereshit Rabba-Fragmenten UB Bremen, VII II, C. 7 und III I C II4a.

I5 Vgl.Jehuda Theodor (Hg.), Bereschit Rabbamit kritischem Apparat und Kommentar, bearbeitetvon Chanokh Albeck, Bd. I, 2. Auflage,Jerusalemı 965, S. 26of.,Zeile 2-Zeile 2.FürMsVatican ebr.6ovgl.nundas OnlineFaksimile Vat.ebr.6o <https://digi.vatlib.it/mss/detail/Vat.ebr.6o> (Zugriff: I8.02.202I). Zu dieser Edition vgl. Tamar Kadari, Minkhah L'Yehudah.JuliusTheodorandtheRedaction of the AggadicMidrashimoftheLand ofIsrael,Jerusalem 2or7 (Hebräisch), S. $30-38$.

16 Vgl. Samuel Krauss, Griechische und lateinische Lehnwörter im Talmud, Midrasch und Targum. Mit Bemerkungen von Immanuel Löw, Bd. 2, Berlin I899, S. I8I.

17 Am linken Spaltenrand steht hier in der Schrift des Schreibers: פי' גיסטריות בעי חליות וצורכי מל[חמה] אני צריך בדיה אני. Vgl. dazu den Kommentar Raschis (ed. Wilna I887, 59d), der in der gedruckten Fassung kürzer ausfällt.

I8 Lehnardt [Anm. I2], S. Iogf.

19 Vgl. Lehnardt [Anm. I2], S. Io9. 